Musées, Patrimoine et Culture scientifiques et techniques

147 | 2013

mai - juin 2013

\title{
Aspects juridiques de la protection des dinosaures
}

Jean-Dominique Wahiche

URL : http://journals.openedition.org/ocim/1230

DOI : 10.4000/ocim. 1230

ISSN : 2108-646X

Éditeur

OCIM

Édition imprimée

Date de publication : 1 mai 2013

Pagination : 13-21

ISSN : 0994-1908

Référence électronique

Jean-Dominique Wahiche, "Aspects juridiques de la protection des dinosaures ", La Lettre de I'OCIM

[En ligne], 147 | 2013, mis en ligne le 01 mai 2015, consulté le 19 avril 2019. URL : http://

journals.openedition.org/ocim/1230; DOI : 10.4000/ocim.1230 


\section{Aspects juridiques de la protection des dinosaures}

\section{Jean-Dominique Wahiche *}

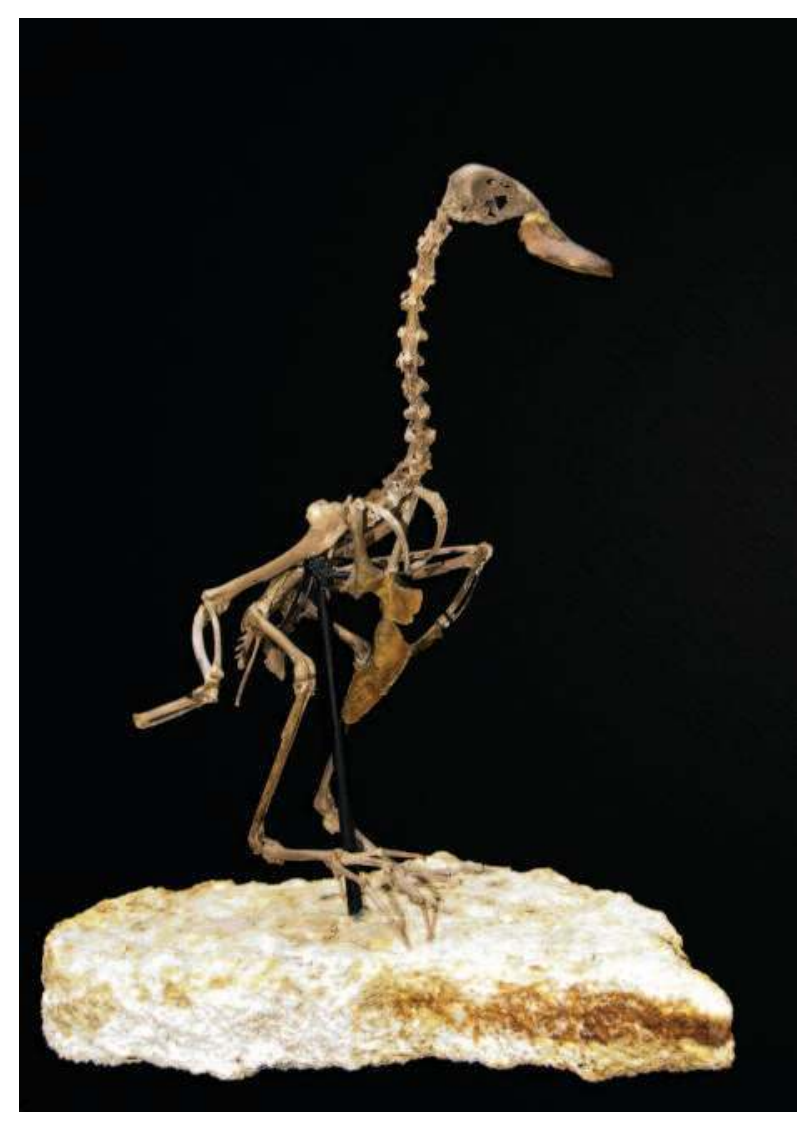

Dendrochen blanchardi, Miocène inférieur de Montaigu-le-Blin (Auvergne), collection particulière F. Escuillié ๑) Muséum d'Orléans/Christophe Camus

* Jean-Dominique Wahiche est docteur d'État en droit, enseignant en droit du patrimoine naturel, département Hommes-Nature-Société, UMR 7206 au Muséum national d'Histoire naturelle wahiche@mnhn.fr
À travers l'examen de tout un arsenal réglementaire national et international, l'auteur montre comment le dinosaure - élément reconnu du patrimoine naturel, scientifique et culturel de l'humanité - est protégé par le droit à tous ces titres, qu'il s'agisse de la protection de l'objet et/ou de la protection de l'espace dans lequel il se trouve

Le titre de cet article cache un régime juridique qui emprunte certains aspects de la protection du patrimoine culturel et s'apparente pour partie à celui du patrimoine biologique. Ceci est compréhensible puisque patrimoine biologique et patrimoine géologique font tous deux partie du patrimoine naturel.

Les restes de dinosaures n'ont plus rien de biologique ; transformés en pierre par le temps et les éléments, ils n'en restent pas moins des témoins étranges et inestimables de l'histoire de la Terre et de la Vie.

Le droit français comme le droit international ont établi des règles visant à préserver les fossiles et en favoriser ainsi l'étude scientifique comme l'éducation du public. Dans un musée, les dinosaures sont protégés au même titre que les autres objets de collections, publiques ou privées, et les dispositions de la Loi n²0025 du 4 janvier 2002 relative aux musées de France leur sont applicables, de même que les autres textes contenus dans le Code du Patrimoine. On abordera ici seulement la situation juridique des fossiles in situ, pour les particularités qu'elle présente.

\section{La protection des spécimens}

La protection des spécimens préhistoriques issus de fouilles a très longtemps concerné les objets façonnés 
de la main de l'homme, autrement dit des objets archéologiques, avant d'embrasser les spécimens naturels présentant un intérêt scientifique. Très rares, ils ne sont pas renouvelables, et leur protection in situ est aussi importante que leur protection ex situ. C'est la convention de l'UNESCO de 1970 qui élève la première au rang d'objet culturel les « objets présentant un intérêt paléontologique». Cette convention interdit le mouvement des biens culturels effectués contrairement aux dispositions règlementaires prises par les États parties, l'adoption de telles dispositions étant recommandée par la convention pour indiquer la disparition de ces objets.

"Aux fins de la présente Convention sont considérés comme biens culturels les biens qui, à titre religieux ou profane, sont désignés par chaque État comme étant d'importance pour l'archéologie, la préhistoire, l'histoire, la littérature, l'art ou la science, et qui appartiennent aux catégories ci-après... Collections et spécimens rares de zoologie, de botanique, de minéralogie et d'anatomie; objets présentant un intérêt paléontologique... ».

Une fois entré dans un musée, le dinosaure est protégé comme tous les autres objets conservés dans un musée et les dispositions de la loi musée et de ses décrets d'application le concernent.

\section{Le statut juridique des fossiles en France}

On s'interrogera sur le statut juridique des fossiles afin de déterminer le régime juridique qui leur sera applicable. Ceci peut être important pour déterminer le droit d'accès et le droit de propriété sur le spécimen.

En droit français, tous les biens sont meubles ou immeubles (Code Civil, art. 516). Ils peuvent être meubles soit par leur nature, soit par détermination de la loi (Code Civil, art. 527). Sont meubles par leur nature les animaux et les corps qui peuvent se transporter d'un lieu à un autre, soit qu'ils se meuvent par eux-mêmes, soit qu'ils ne puissent changer de place que par l'effet d'une force étrangère (Code Civil, art. 528). En revanche, «les fonds de terre et les bâtiments sont immeubles par leur nature» (Code Civil, art. 518). En pratique, le statut du fossile va donc changer, selon qu'il est attaché au sol ou qu'il en est détaché. Ceci a été établi par un arrêt du Tribunal de Grande Instance de Millau (TGI Millau, 26 mai 1988, Mme Bichet contre Commune de Tournemire et autre). Des vertèbres fossiles de Plésiosaure ont été qualifiées d'immeubles car elles n'étaient pas détachées du sol par l'effet de l'érosion mais se trouvaient encore incluses dans la gangue marneuse.

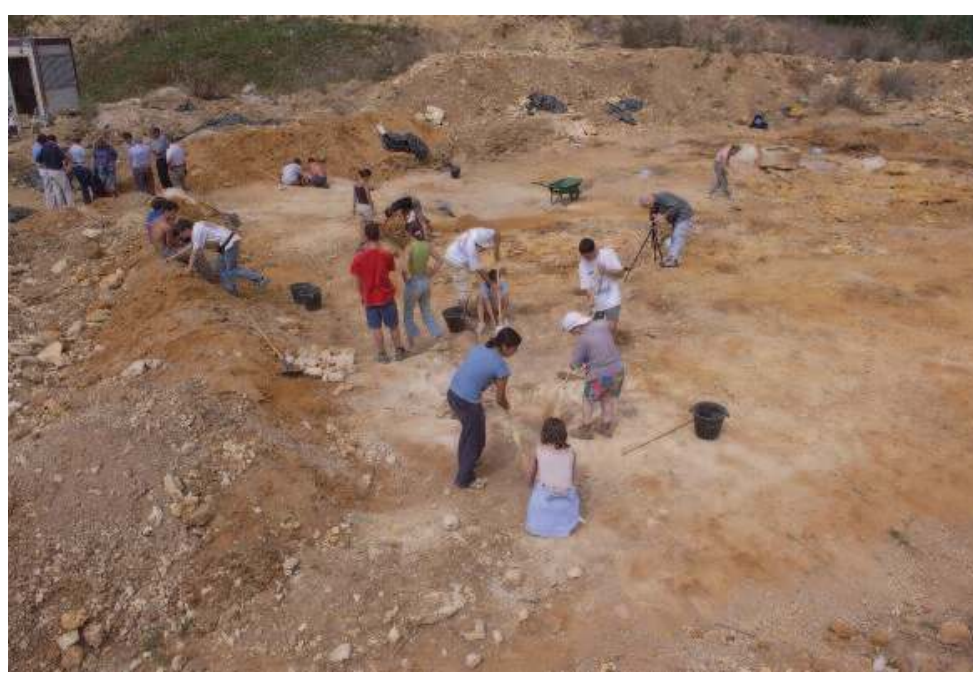

Chantier de fouilles à Montréal-du-Gers (c) Francis Duranthon

Concernant la propriété du fossile, la distinction n'aura pas d'effet, car «la propriété du sol emporte la propriété du dessus et du dessous. Le propriétaire peut faire au-dessus toutes les plantations et constructions qu'il juge à propos...Il peut faire au-dessous toutes les constructions et fouilles qu'il jugera à propos, et tirer de ces fouilles tous les produits qu'elles pewvent fournir, sauf les modifications résultant des lois et règlements relatifs aux mines, et des lois et règlements de police » (Code Civil, art. 552).

On peut aussi s'interroger sur la possible qualification de « trésor » des restes fossiles d'un dinosaure. L'article 716 du Code Civil définit le trésor comme " toute chose cachée ou enfouie sur laquelle personne ne peut justifier sa propriété, et qui est découverte par le pur effet du hasard». Ceci est important car «la propriété d'un trésor appartient à celui qui le trouve dans son propre fonds; si le trésor est trouvé dans le fonds d'autrui, il appartient pour moitié à celui qui l'a découvert, et pour l'autre moitié au propriétaire du fonds ». Les termes «cachée » et « enfouie » supposent toutefois une intervention humaine, ce qui n'est pas le cas pour les fossiles qui se trouvent là où ils sont par le seul effet de la nature et de ses éléments. Autre conséquence, les dispositions de l'article L 531 du Code du Patrimoine ne semblent pas être applicables. Celui-ci prévoit que «l'autorité administrative statue sur les mesures définitives à prendre à l'égard des déconvertes de caractère immobilier faites fortuitement. Elle peut, à cet effet, ouvrir pour ces vestiges une instance de classement conformément à la législation sur les monuments historiques. Les déconvertes de caractère mobilier faites fortuitement sont confiées à l'État pendant le délai nécessaire à leur étude 
Le régime juridique de l'acquisition des collections de paléontologie reste embryonnaire, sans véritable contrôle. Lorsquil s'agit de l'entrée d'une collection dans un établissement dépendant d'une collectivité publique, la prudence est toujours de rigueur en la matière afin d'éviter que l'établissement ne puisse être considéré comme receleur aux yeux de la loi.

\section{La question des spécimens d'origine française}

Pour ces spécimens, le seul droit qui s'applique est le droit de propriété. En matière d'objets meubles, comme le sont les fossiles, possession vaut titre de propriété. Notons que d'un point de vue juridique strict, ce sont des objets meubles par destination, c'est-à-dire qu'ils deviennent meubles lorsqu'ils sont détachés du terrain lors d'une opération de fouille. Concrètement, le statut de meuble signifie que le spécimen est la propriété de celui qui le détient. Dans le cadre d'un achat pour les collections d'un musée, il convient toutefois de s'assurer de certains points :

1/ Que le vendeur ou donateur est bien le propriétaire légitime de l'objet. Ce point est toujours délicat. Lors de la fouille d'un site, il faut obtenir une autorisation écrite du propriétaire qui doit préciser la dévolution des objets récoltés. Ce document doit être produit pour les spécimens issus de fouilles récentes. Notons que pour les récoltes de surface ce document est quasi impossible à obtenir. Quant aux récoltes anciennes, on se bornera en général à la bonne foi du vendeur ou du donateur mais il est assez simple de vérifier la date de découverte d'un site par les publications qui lui sont en général liées et donc de vérifier les dires du vendeur. 2/ N'étant pas et ne pouvant pas constituer un trésor (au sens de la loi sur les objets archéologiques), l'objet paléontologique échappe aux règles de découverte et de partage y afférent et ce n'est que dans de rares circonstances qu'il peut constituer un objet paléontologique dont les fouilles et prélèvements sont réglementés. L'objet paléontologique n'a pas à l'heure actuelle de statut propre encadré par des textes législatifs, faute de liste de spécimens paléontologiques protégés. Il bénéficie seulement du régime du lieu dans lequel il est découvert et de la réglementation qui s'y applique. Celle-ci peut souffrir plus ou moins de restrictions liées au statut du lieu, lorsque le fossile se situe sur des espaces bénéficiant d'un régime de protection juridique (parc national, réserve naturelle, site classé ou inscrit, propriété privée ou publique). Dans le cas des sites relevant des lois sur la protection de la nature (parcs nationaux, réserves), des autorisations de fouille ou des preuves matérielles d'une récolte antérieure aux lois de 1976 sont à réclamer au vendeur ou donateur. Il en va de même pour les objets provenant de sites classés, il faut obtenir du vendeur ou donateur l'autorisation de fouille délivrée par les services de l'État.

\section{Les spécimens originaires de l'étranger}

Les fossiles font l'objet d'un intense commerce international. De nombreux exemples récents (dinosaures mongol et chinois commercialisés aux États-Unis...) montrent quil faut être très prudent dans ces cas là puisque ils ont donné lieu à des contentieux internationaux. La Chine a par exemple obtenu la restitution de nombreux spécimens achetés par des musées américains. L'état mongol a poursuivi un vendeur de fossiles aux États-Unis.

Sauf cas particulier, la traçabilité des spécimens paléontologiques n'est pas des plus évidentes. Le contrôle de leur commercialisation reste aléatoire, subordonné au régime des ventes au déballage dans le cadre de manifestations d'amateurs de minéraux et de fossiles ou à celui de commerces plus établis, faute de prescriptions spécifiques tant en ce qui concerne les objets recueillis sur le territoire national que ceux qui y ont été importés. En tout état de cause, l'acheteur doit obtenir du vendeur des certificats d'importation et d'exportation en bonne et due forme, émis par les services douaniers à la sortie du pays concerné et à l'entrée des spécimens sur le territoire national. Il convient toutefois, afin de vérifier que les documents douaniers d'exportation ont bien été obtenus de manière régulière, de s'assurer que les législations locales permettent bien la récolte et la commercialisation de fossiles auprès des services de l'ambassade du pays concerné.

\section{Les spécimens recueillis dans le cadre d'une activité professionnelle}

Il faut, enfin, composer avec le régime des collections constituées par des agents publics dans le cadre de missions scientifiques ou d'actions d'enseignement et qui sont déposées dans des établissements d'enseignement et de recherche. Le propriétaire légitime est l'organisme qui paye les collecteurs et non les chercheurs comme ceux-ci le croient très souvent. C'est le cas du CNRS, des universités, des laboratoires de recherche. Toute demande de dépôt, de vente ou de don d'une collection de chercheur doit être réglée au cas par cas avec son organisme payeur. Par principe, la vente d'une telle collection ne peut être retenue puisqu'elle a été constituée avec de l'argent public.

De manière générale, l'absence de réglementation particulière invite en tout cas à rechercher dans les exemples étrangers des éléments qui pourraient être repris en droit interne et proposés à nos élus pour faire évoluer la législation en la matière.

Francis Duranthon

Conservateur en chef,

Directeur du muséum d'Histoire naturelle de Toulouse 
scientifique. Au terme de ce délai, qui ne peut excéder cinq ans, leur propriété demeure réglée par l'article 716 du Code Civil. Toutefois, l'État peut revendiquer ces découvertes moyennant une indemnité fixée à l'amiable ou à dire d'experts. Le montant de l'indemnité est réparti entre l'inventeur et le propriétaire, suivant les règles $d u$ droit commun, les frais d'expertise étant imputés sur elle. Dans un délai de deux mois à compter de la fixation de la valeur de l'objet, l'Etat peut renoncer à l'achat. Il reste tenu, en ce cas, des frais d'expertise ».

La propriété du dinosaure revient donc au propriétaire du sol, d'autant que le Code Civil précise encore que «la propriété d'une chose soit mobilière, soit immobilière, donne droit sur tout ce qu'elle produit, et sur ce qui s'y unit accessoirement soit naturellement, soit artificiellement » (Code Civil, art. 546).

La seule solution donc pour accéder aux fossiles que l'on suppose exister sur un terrain est d'en demander l'accès au propriétaire. Les conséquences de cette autorisation d'accès doivent toutefois être étudiées. Cette autorisation peut être qualifiée de tolérance, qui devra pouvoir être prouvée. En outre, «les actes de pure faculté et ceux de simple tolérance ne peuvent fonder ni prescription, ni possession " (Code Civil, art. 2262), ce qui signifie que l'autorisation d'accès n'entraîne pas, pour le découvreur (l'inventeur), la propriété des spécimens qu'il aura découverts.

La solution la plus appropriée est sans nul doute d'établir un contrat écrit, fixant les modalités d'accès et surtout déterminant la propriété des découvertes et le cas échéant les règles de partage des avantages issus des découvertes. Cet accord devra être recherché même dans le cas de terrains non clôturés, car l'absence de clôture ne signifie pas le libre accès. En France, il n'y a guère de terrains qui n'appartiennent à personne : ils sont soit privés, soit publics (communaux, domaniaux...) mais relèvent toujours d'un propriétaire auquel il faudra donc s'adresser pour entreprendre une fouille. Le fossile a donc toujours un propriétaire. On peut d'ailleurs préciser que, par accord entre le propriétaire et le fouilleur, le fossile dans sa gangue peut devenir meuble par anticipation, catégorie d'origine jurisprudentielle : les meubles par anticipation sont des immeubles qui sont considérés comme étant des meubles en anticipant leur état prochain. L'exemple habituel est celui de la vente de récoltes sur pied : celles-ci sont considérées par les tribunaux comme étant des biens meubles, car c'est la récolte détachée du sol qui est l'objet du contrat de vente (CA Montpellier, 23 juin 1927).

\section{La protection internationale}

La Convention concernant les mesures à prendre pour interdire et empêcher l'importation, l'exportation et le transfert de propriétés illicites des biens culturels (UNESCO, Paris, 14 novembre 1970), assimile le fossile aux biens culturels afin de le protéger. La protection ici ne concerne pas l'accès mais le mouvement du fossile en tant que meuble ; on peut rapprocher ce dispositif de celui institué par la Convention sur le commerce international des espèces de faune et de flore sauvages menacées d'extinction (Washington, 3 mars 1973, communément appelée CITES) qui, afin de protéger les espèces en danger déterminées par une liste régulièrement mise à jour, interdit leur mouvement et leur commerce.

Selon la Convention de Paris, sont illicites l'importation, l'exportation et le transfert de propriété des biens culturels, effectués contrairement aux dispositions prises par les États parties à cet accord. Ceci laisse aux États membres la liberté de déterminer ce qui est ou n'est pas illicite, selon leur propre législation. Comme dans tout traité international, les dispositions de l'accord ne concernent que les États parties (actuellement, 122 États sont parties à la convention) et ne s'appliquent qu'après son entrée en vigueur. Elles ne concernent que les objets de provenance illicite (volés, ou exportés illicitement), car il ne s'agit pas ici d'interdire le commerce licite de biens culturels.

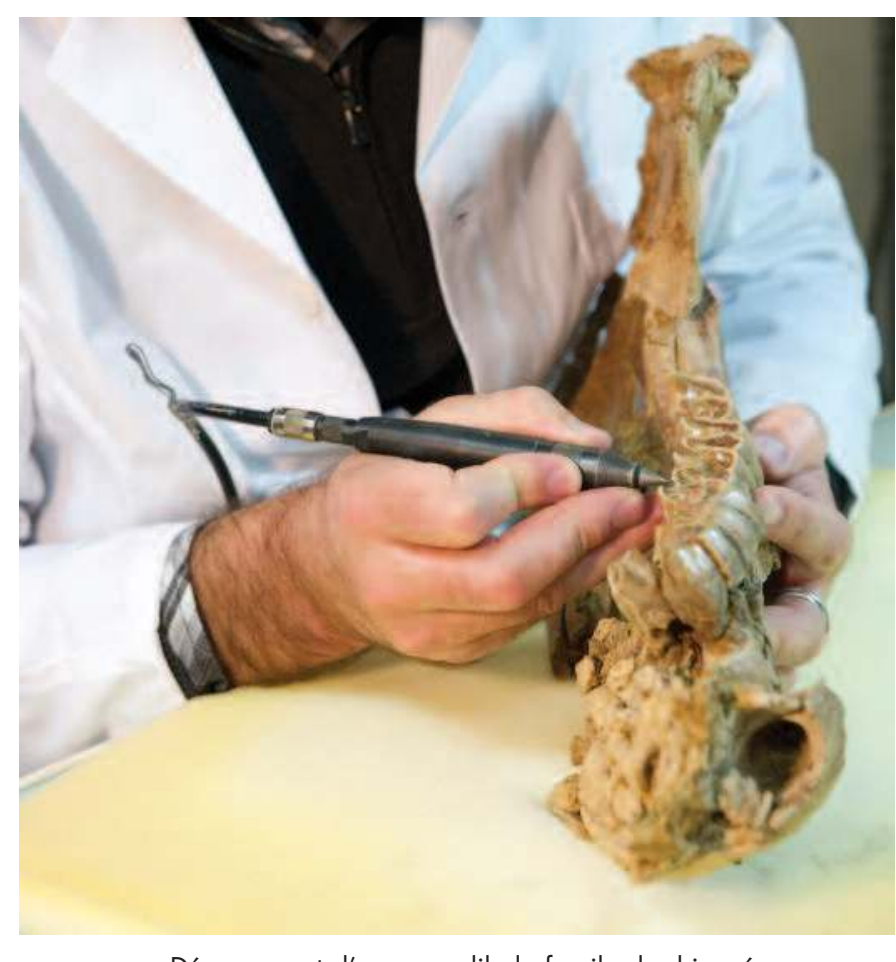

Dégagement d'une mandibule fossile de rhinocéros (c) Christian Nitard 
Cette convention protège les intérêts de tout propriétaire dépossédé ; cela peut être un individu, une personne morale ou un État.

Elle intervient tant en matière de prévention qu'en matière de restitution : la prévention est prévue par le biais de la création de services nationaux spécifiques, l'adoption de mesures légales ou administratives, l'introduction d'un certificat d'exportation approprié, l'obligation pour les marchands d'art de tenir un registre relatif à l'origine des objets ou la sensibilisation du public par le biais de mesures de formation.

La restitution est prévue notamment par l'admission des actions en restitution des objets volés, et la coopération visant à faciliter la restitution des objets illicitement exportés (si ceci est compatible avec le droit de l'État Partie concerné).

La Convention de l'UNESCO de 1970 privilégie la coopération inter-étatique et opère principalement par la voie diplomatique ainsi que par voie judiciaire (dans la mesure encore où ceci est admis par le droit de l'État partie concerné).

Il est intéressant de noter que tant les États parties par le biais de la voie diplomatique que les particuliers ou les personnes morales - d'après la loi de l'État partie - peuvent demander la restitution.

Pour mettre en œuvre ces principes, les États s'engagent à instituer un certificat approprié par lequel l'État exportateur spécifie que l'exportation du ou des biens culturels visés est autorisée par lui, ce certificat devant accompagner le ou les biens culturels régulièrement exportés et corrélativement à interdire la sortie de leur territoire des biens culturels non accompagnés du certificat d'exportation visé. De même, ils doivent prendre toutes les mesures nécessaires, conformes à la législation nationale, pour empêcher l'acquisition, par les musées et autres institutions similaires situés sur leur territoire, de biens culturels en provenance d'un autre État partie à la Convention, biens qui auraient été exportés illicitement après l'entrée en vigueur de la Convention.

La Convention sort du cadre strict de l'interdiction du commerce illicite pour prévenir les acquisitions frauduleuses en protégeant l'accès aux sites : elle confie aux États le soin de contrôler des fouilles archéologiques, assurer la conservation in situ de certains biens culturels et protéger certaines zones réservées à des recherches archéologiques futures. Certes, il est question de sites archéologiques, et non pas géologiques ou paléontologiques, mais ceci constitue un premier pas, qui sera développé ci-après.

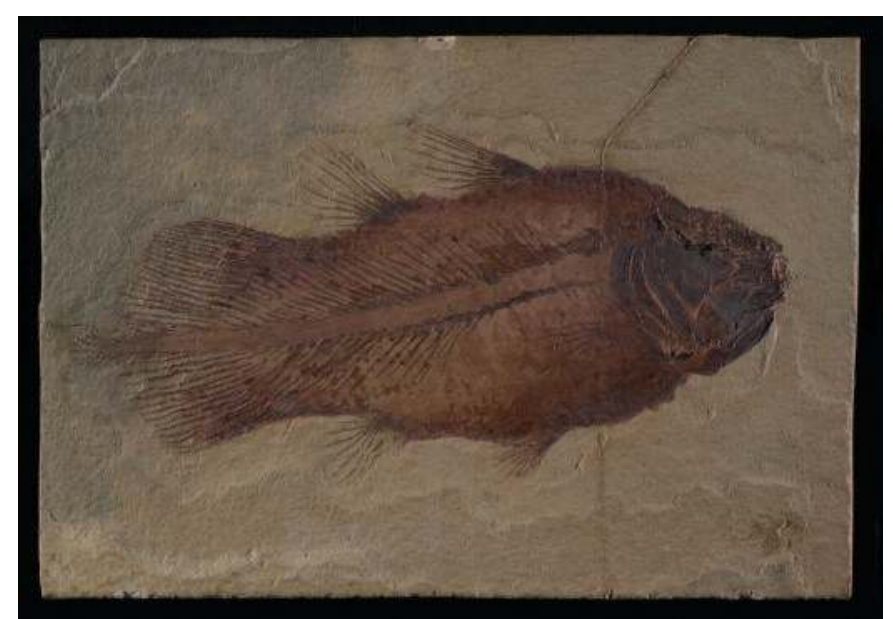

Fossile de Hadronector donbirdi (Cœlacanthe) (c) Frédéric Ripoll

\section{La protection des sites}

\section{La protection nationale}

Pour protéger le dinosaure dans son environnement, la meilleure solution est de protéger le site qui en renferme les restes. Cette méthode a l'avantage pratique de protéger ipso facto les spécimens et surtout de conserver l'environnement fossilifère pour des fouilles ultérieures.

La loi du 22 juillet 1960 relative à la création de Parcs nationaux mentionne ainsi la conservation du sous-sol pour le protéger des dégradations naturelles ou artificielles, et son décret d'application prévoit des peines d'amendes et d'emprisonnement pour ceux qui auront, sans autorisation, prélevé des minéraux ou des fossiles dans un Parc national ou qui les auront "détenus, transportés, colportés, mis en vente, vendus ou achetés sciemment », expression que l'on retrouvera en 1976 dans la loi relative à la protection de la nature, issue elle-même de la Convention de Washington déjà mentionnée. Celle-ci va plus loin, sort du cadre des parcs et, plus généralement, interdit « la destruction des sites contenant des fossiles permettant d'étudier l'histoire du monde vivant... » (art. 3). Elle prévoit le classement en réserve naturelle de parties du territoire lorsque la conservation de fossiles revêt une importance particulière (art. 16), notamment " pour l'étude de l'évolution de la vie...».

Le Code de l'Environnement (L. 411-1 et L. 411-2) qui reprend les dispositions de cette loi prévoit l'établissement d'une liste des «sites contenant des fossiles permettant d'étudier l'histoire du monde vivant ainsi que les premières activités humaines ». La destruction de ces sites, et la destruction ou l'enlèvement des fossiles 
La convention de l'UNESCO concernant les mesures à prendre pour interdire et empêcher l'importation, l'exportation et le transfert de propriété illicite de biens culturels a été signée par la Fédération de Russie le 28 avril 1988 et par la France le 7 janvier 1997. Si la compréhension du texte ne pose pas de problème pour le personnel des muséums, son application concrète est plus délicate. Les modalités douanières sont internationales et destinées essentiellement à la perception des taxes. Ces documents douaniers sont donc indispensables, mais pas suffisants. Pour le montrer, je donnerai comme exemple des acquisitions paléontologiques venant de la Fédération de Russie. Cependant, afin de ne pas donner des idées à des vendeurs peu scrupuleux, je ne ferai pas de commentaires sur les points particuliers qu'il convient de vérifier et de croiser.

Les documents douaniers devraient être fournis et faire l'objet d'un examen critique lors d'une acquisition même dans une salle des ventes. Malheureusement, cela est rarement possible, à ma connaissance cela a été fait pour une seule vente en France, pourtant c'est une pratique commune pour les ventes en entomologie, ornithologie... Il n'y a donc rien d'impossible.

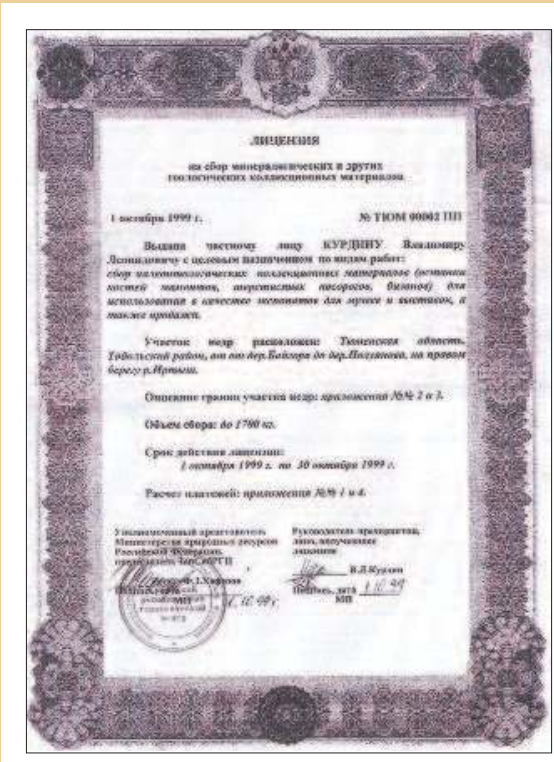

\section{La licence}

Sur la collection des matériaux minéraux et autres matériaux géologiques. - Date ........

- Licence a été délivrée au particulier ...... ; nom et prénom

- Avec les objectifs spéciaux : collections des matériaux paléontologiques (restes des os de mammouth, de rhinocéros laineux, de bisons) leur utilisation en qualité de pièces de musée et d'exposition, leur vente.

- Le terrain de recherche : ...... région village ........

- La description des limites du territoire de recherche : annexe 2,3

- Le volume de collection : ..... maximum ..... kg

- Durée de la licence : du .......... au ...........

- Règlement des comptes : annexes N1 et N4

- Le représentant mandaté du

- Ministère des Ressources Naturelles,

- Fédération de Russie.

- Date et signature.

(traduction non contractuelle)

Les informations sont à croiser avec celles du certificat. Souvent, le vendeur qui fait l'exportation n'est pas le collecteur. \|l est souhaitable d'avoir une attestation ou une copie de la transaction entre le récolteur et l'exportateur s'ils sont différents. À noter que la collecte est limitée dans le temps et dans l'espace. Elle est spécifique d'un groupe paléontologique. (c) G.Pacaud

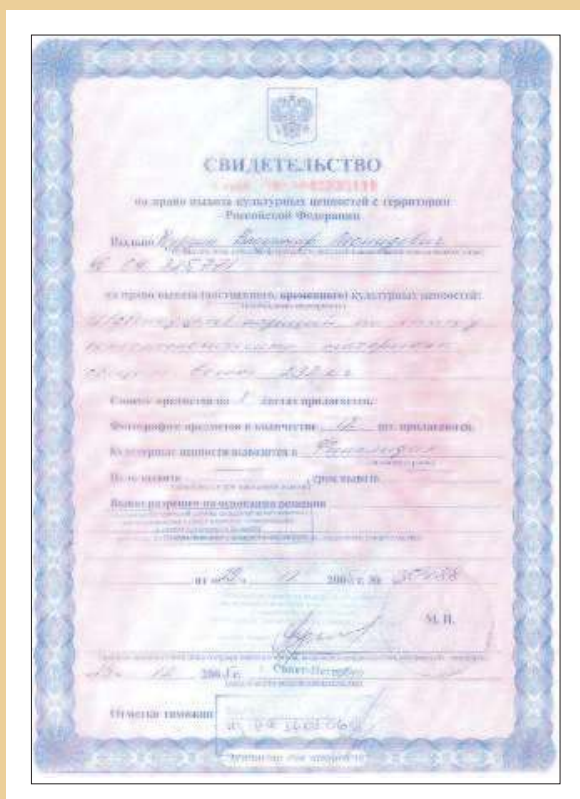

\section{Le certificat d'exportation}

Certificat d'exportation.

$N^{0} \ldots . . .$.

Droit d'exporter des biens culturels du territoire russe (Fédération de Russie)

- Certificat a été délivré ...... nom, prénom d'une personne physique ou civile avec le droit d'exporter (permanent, temporaire, la décision est soulignée et l'autre rayée) les objets de valeur culturelle : ....... désignation et poids .......

- La liste des objets est annexée : ..... nombre de feuilles.

- Les photos des objets sont annexées : ..... nombre de photos.

- Les objets vont être exportés : ..... nom du pays .....

- Le but d'exportation ......... les délais ........

- Autorisation : ..... organisme d'État délivrant le certificat ............

- Date .......... $n^{\circ} \ldots \ldots . .$.

- Nom et prénom du fonctionnaire délivrant le certificat.

- Date et lieu.

- Les notes de la dovane

(traduction non contractuelle)

Dans cet exemple, il s'agit d'une exportation permanente de 12 objets de la liste, de matériaux paléontologiques, le poids est de $232 \mathrm{~kg}$. II y a 12 photographies. () G.Pacaud 


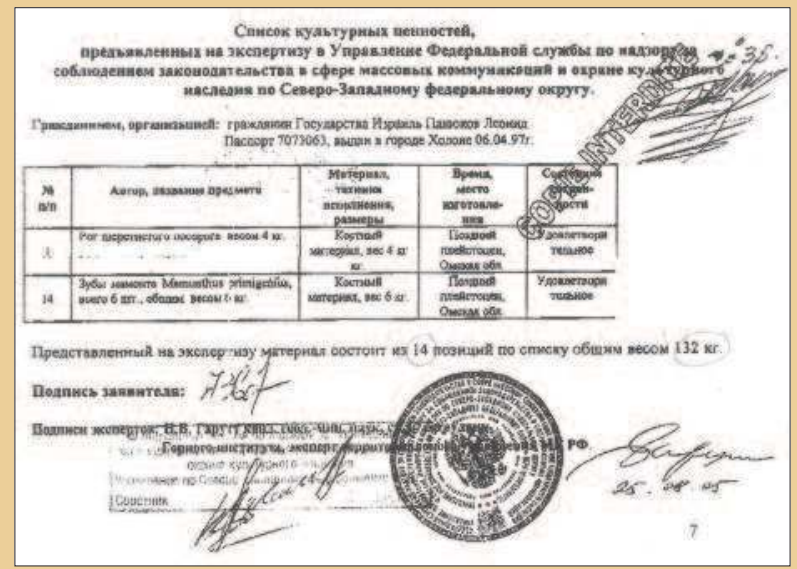

Annexe au certificat d'exportation : liste des spécimens à exporter (reproduction partielle) () G.Pacaud

Seul l'exemple de la Russie est donné ici. Si l'esprit de la convention est respecté par les signataires son application concrète varie d'un pays à l'autre, mais aussi dans le temps. Ainsi, avant janvier 2011, le commerce de fossiles était possible en Chine, mais l'exportation interdite, donc une situation simple à appliquer. Maintenant, les fossiles chinois sont classés au point de vue vente en 3 catégories : interdiction ou sous condition jusqu'à la vente libre avec ou sans exportation.

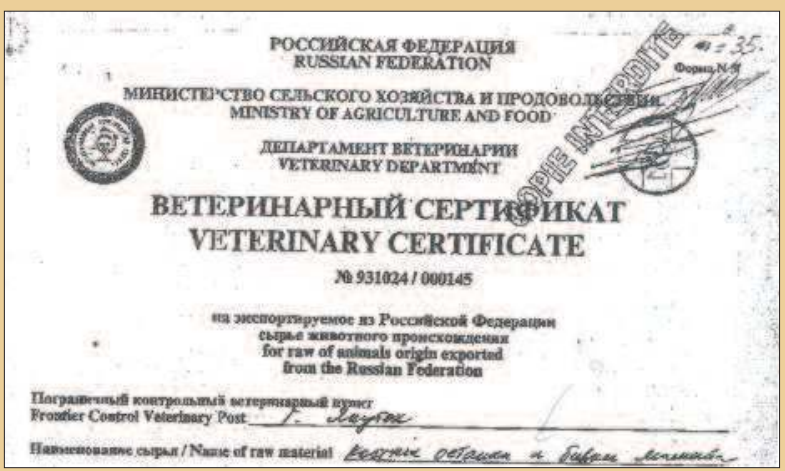

Annexe au certificat d'exportation (certificat vétérinaire) : ce document est important pour certains spécimens (le bébé mammouth « Kroma » exposé en France était contaminé par l'anthrax). Ce certificat est indépendant du document d'accompagnement français indiquant que l'importateur ne destine pas ces produits à l'alimentation humaine ou animale (reproduction partielle). () G.Pacaud

Il est à noter que les musées doivent redoubler de vigilance sur l'examen des documents d'accompagnement pour certaines sorties de spécimens paléontologiques du territoire de la Fédération de Russie, notamment en ce qui concerne les sorties par SaintPétersbourg et Blagovechtchensk ou via la Finlande, l'Estonie...

Gilles Pacaud

Conservateur en chef honoraire présents sur ces sites sont interdits. Larticle L. 342-1 permet au ministre de l'Environnement de prendre un arrêté fixant la liste des sites « en raison de leur importance pour la compréhension de l'histoire de la terre et de l'utilisation de ressources naturelles par l'homme (...) lorsqu'un intérêt scientifique particulier ou que les nécessités de la préservation du patrimoine minéralogique le justifient ».

Le classement et la protection de parties de territoire nécessitent qu'un inventaire des sites qui font partie $\mathrm{du}$ " patrimoine commun de la nation» (art. 200-1 du nouveau Code rural) soit établi. Une fois cette mesure prise, il sera interdit de détruire et enlever les fossiles présents sur ces sites (art. 211-1 du nouveau Code rural). Le Code de l'Environnement vient renforcer cette nécessité d'établir un inventaire, cette fois $\mathrm{du}$ « patrimoine naturel pour les richesses écologiques, faunistiques, floristiques, géologiques, minéralogiques et paléontologiques».

Ces inventaires sont établis sous la responsabilité du Muséum national d'Histoire naturelle (art. L.411-5 du Code de l'Environnement).

Il existe toutefois des différences entre les régimes de protection, et le fouilleur est bien inspiré en se renseignant sur la législation applicable localement.
Ainsi, le périmètre de protection institué autour des dix-neuf sites de la Réserve naturelle géologique des Alpes de Haute-Provence concerne l'ensemble du territoire de quarante-sept communes : est exclusivement interdite la recherche et la collecte de minéraux et fossiles, mais la simple «collecte » de pièces dégagées par l'érosion est autorisée (articles L. 332-16 à 18 du Code de l'Environnement et arrêté no 89-527 du 15 mars 1989 du préfet des Alpes de Haute-Provence, complété et étendu par les arrêtés du 18 septembre 1992, 17 décembre 1996 et 12 juin 1998).

On notera que parmi les milieux susceptibles de bénéficier de la protection de l'article L. 146-6 du Code de l'Urbanisme relatif aux sites ou aux paysages remarquables ou caractéristiques du patrimoine naturel et culturel du littoral, sont concernées les formations géologiques telles que les gisements de minéraux ou de fossiles, les stratotypes, les grottes ou les accidents géologiques remarquables (art. R. 146-1 du Code de l'Urbanisme).

\section{La protection internationale}

La Convention pour la protection du patrimoine mondial, culturel et naturel (UNESCO, Paris, 1972), qui compte 195 États membres et 8 associés, a pour objet de protéger les sites remarquables tels que : 
- les monuments naturels constitués par des formations physiques et biologiques ou par des groupes de telles formations qui ont une valeur universelle exceptionnelle du point de vue esthétique ou scientifique; - les formations géologiques et physiographiques et les zones strictement délimitées constituant l'habitat d'espèces animales et végétales menacées, qui ont une valeur universelle exceptionnelle du point de vue de la science ou de la conservation ;

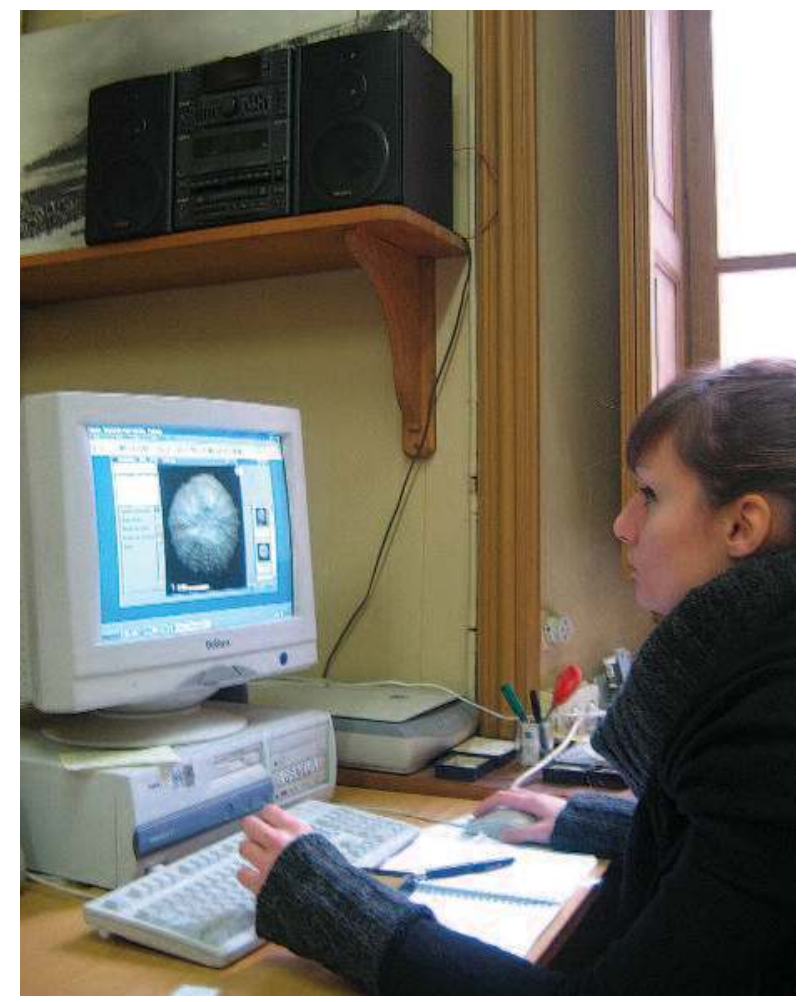

Inventaire de collections sous SN Base (C) Francis Duranthon
- les sites naturels ou les zones naturelles strictement délimitées, qui ont une valeur universelle exceptionnelle du point de vue de la science, de la conservation ou de la beauté naturelle (art. 2).

Les sites renfermant des fossiles peuvent donc être protégés au titre de l'intérêt scientifique qu'ils présentent. Chaque État partie doit délimiter les différents sites situés sur son territoire. Comme pour la Convention de 1970 déjà citée, des mesures préventives doivent être mises en place afin d'identifier les sites et de les protéger. Politique générale, recherche scientifique et création de structures de protection, ainsi qu'un dispositif juridique approprié sont les différentes voies recommandées par la Convention (art. 5).

La Convention a également institué un Comité intergouvernemental de la protection du patrimoine culturel et naturel de valeur universelle exceptionnelle dénommé « le Comité du patrimoine mondial ». Sur la base des inventaires soumis par les États, le Comité établit, met à jour et diffuse, sous le nom de « liste du patrimoine mondial », une liste des biens du patrimoine culturel et du patrimoine naturel, tels qu'ils sont définis aux articles 1 et 2 de la Convention, qu'il considère comme ayant une valeur universelle exceptionnelle en application des critères qu'il aura établis. Les sites ainsi classés peuvent bénéficier de moyens techniques et financiers exceptionnels attribués par le Comité ainsi que de la collaboration des autres États membres.

Ainsi, les sites fossilifères de Riversleigh et Naracoorte, en Australie, de Chengjang en Chine ou de Messel en Allemagne ont été classés à ce titre.

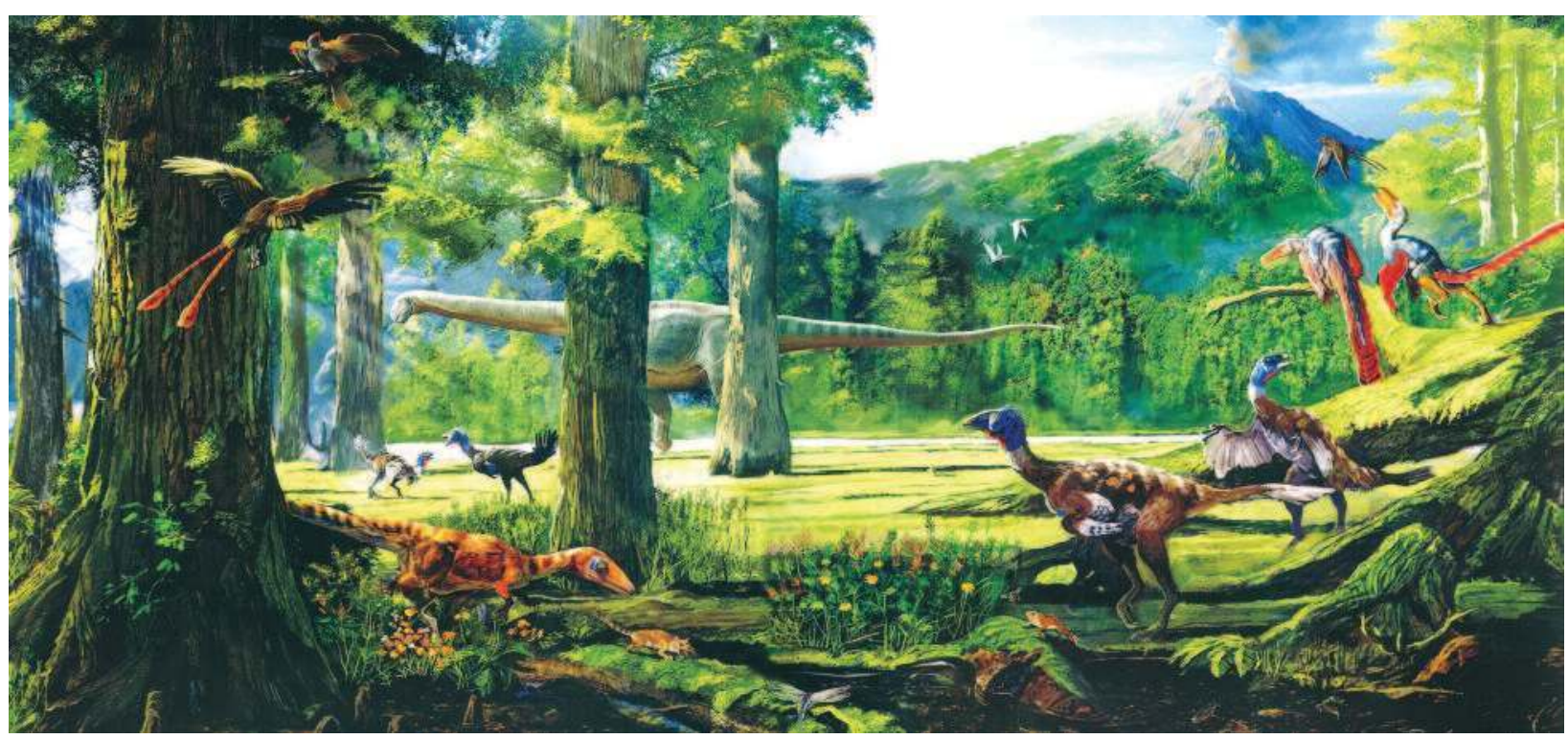

La faune de Jehol (Chine) au Crétacé inférieur, musée Paléontologique du Liaoning ๑) Muséum d'Orléans/Christophe Camus 


\section{Conclusion}

Comme pour tous les régimes de protection de la nature, les dinosaures sont " protégés » en tant qu'espèce et par la protection de l'espace dans lequel on trouve leurs restes. L'appropriation reste possible, mais elle est encadrée par une réglementation stricte, sans toutefois que celle-ci crée d'exception au régime de la propriété privée. Pour qu'il en soit autrement, il faudrait que s'applique un dispositif tel que celui de la CITES interdisant la vente, l'achat, l'échange, le colportage de tout fossile de vertébrés, ce qui n'est pas nécessairement souhaitable si l'on considère l'intérêt limité de certains fragments.

Le dinosaure apparaît donc bien protégé, dans le musée comme hors du musée. Inventorié, classé, expertisé, étudié, exposé, cette vedette des muséums d'Histoire naturelle est un élément reconnu du patrimoine des nations qui les abritent, naturel par son origine, scientifique par son témoignage, culturel par sa dimension légendaire.

Le droit le protège donc à tous ces titres.

\section{Sources}

Convention concernant les mesures à prendre pour interdire et empêcher l'importation, l'exportation et le transfert de propriétés illicites des biens culturels. Paris : UNESCO, 14 novembre 1970.

Convention pour la protection du patrimoine mondial, culturel et naturel. Paris : UNESCO, 1972

Code de l'Environnement, notamment les articles L. 332 et L. 411

Code rural, notamment les articles L. 211 et L. 242

Loi 60-708 du 22 juillet 1960 relative à la création de parcs nationaux

Loi 76-629 du 10 juillet 1976 relative à la protection de la nature

Loi 95-101 du 2 février 1995 relative au renforcement de la protection de l'environnement

Loi 2002-276 du 27 février 2002 relative à la démocratie de proximité

Loi du 14 avril 2006 relative aux parcs nationaux, aux parcs naturels marins et aux parcs naturels régionaux 